\title{
Competencias laborales de los pedagogos egresados
}

\author{
Work skills of graduate educators \\ Habilidades de trabalho de educadores de pós-graduação
}

Recibido: 20/01/2021 | Revisado: 20/01/2021 | Acepto: 23/01/2021 | Publicado: 30/01/2021

\author{
María Luisa Belmonte \\ ORCID: https://orcid.org/0000-0002-1475-3690 \\ Universidad de Murcia, España \\ E-mail: maríaluisa.belmonte@um.es \\ José Santiago Álvarez-Muñoz \\ ORCID: https://orcid.org/0000-0002-9740-6175 \\ Universidad de Murcia, España \\ E-mail: josesantiago.alvarez@um.es \\ Abraham Bernárdez-Gómez \\ ORCID: https://orcid.org/0000-0003-1862-5554 \\ Universidad de Murcia, España \\ E-mail: abraham.bernardez@um.es
}

\begin{abstract}
Resumen
Hace apenas unos años, dentro del contexto de globalización económica del pasado siglo XX y durante la revolución tecnológica, las organizaciones e instituciones junto con el concepto de trabajo en sí, se han ido reconfigurando, y lógicamente, el perfil de competencias exigido a jóvenes y trabajadores, también. La sociedad, progresivamente con el cambio, va requiriendo profesionales competentes en ámbitos cada vez más trasversales, y nos encontramos ante la necesidad de poseer altas capacidades de adaptación y actualización. Se reclaman personas versátiles, capaces no solo en el círculo cerrado de su ámbito laboral, sino con actitudes de trabajo en grupo, capacidad de adaptación, flexibilidad, entre otros. Este proyecto de intervención surge de la necesidad de dotar a los jóvenes de competencias laborales transversales que actualmente se requieren en el mercado profesional. Las competencias que más protagonismo adquieren son algunas como el trabajo en equipo y las habilidades sociales, siendo competencias imprescindibles a desarrollar por un pedagogo. Los destinatarios a los que va dirigido son los alumnos recién graduados en pedagogía por la Universidad de Murcia. Se llevará a cabo en el Servicio Regional de Empleo y Formación, ya que va destinado a esclarecer el futuro profesional de los jóvenes, que atraviesa una situación crítica en nuestro país. A través de una serie de actividades se potenciarán esas competencias, con el fin de impulsar la autonomía para la búsqueda activa de empleo. Así, se espera fomentar la búsqueda fructuosa de empleo de los pedagogos recién egresados.
\end{abstract}

Palabras clave: Competencias laborales; Trabajo en equipo; Habilidades sociales; Pedagogo.

\begin{abstract}
Just a few years ago, within the context of economic globalization of the last 20th century and during the technological revolution, organizations and institutions, together with the concept of work itself, have logically reconfigured the profile of skills required of young people and workers, too. Society, progressively with the change, is requiring competent professionals in increasingly transversal fields, and we find ourselves faced with the need to possess high capacities for adaptation and updating. Versatile people are called for, capable not only in the closed circle of their work environment, but also with group work attitudes, adaptability, flexibility, among others. This intervention project arises from the need to provide young people with transversal job skills that are currently required in the professional market. The competences that acquire the most prominence in this intervention project are some such as teamwork and social skills, being essential competences to be developed by a pedagogue. The recipients of this project are recent graduates in pedagogy from the University of Murcia. It will take place at the Regional Employment and Training Service, as it is aimed at clarifying the professional future of young people, who are going through a critical situation in our country. Through a series of activities, these competences will be enhanced, in order to promote autonomy for the active search for employment. Thus, it is hoped to promote the fruitful search for employment of recently graduated pedagogues.
\end{abstract}

Keywords: Job skills; Teamwork; Social skills; Pedagogue.

\section{Resumo}

Há poucos anos, no contexto da globalização econômica do século XX e durante a revolução tecnológica, organizações e instituições, juntamente com o conceito de trabalho em si, vêm reconfigurando, e logicamente, o perfil de habilidades exigido dos jovens e trabalhadores, também. A sociedade, progressivamente com a mudança, está 
exigindo profissionais competentes em campos cada vez mais transversais, e estamos diante da necessidade de alta adaptabilidade e atualização. Pessoas versáteis são demandadas, capazes não apenas no círculo fechado de seu campo de trabalho, mas com atitudes de trabalho em grupo, adaptabilidade, flexibilidade, entre outras. Essa minuta de intervenção decorre da necessidade de proporcionar aos jovens habilidades de trabalho transversais que atualmente são exigidas no mercado profissional. As habilidades que mais se destacou neste projeto de intervenção são algumas como trabalho em equipe e habilidades sociais, sendo competências essenciais a serem desenvolvidas por um pedagogo. Os beneficiários do projeto são os recém-formados em pedagogia pela Universidade de Múrcia. Será realizado no Serviço Regional de Emprego e Formação, pois pretende esclarecer o futuro profissional dos jovens, que está em situação crítica em nosso país. Essas habilidades serão aprimoradas por meio de uma série de atividades, a fim de aumentar a autonomia para a busca ativa pelo emprego. Assim, espera-se incentivar a busca frutífera pelo emprego de educadores recém-formados.

Palavras-chave: Habilidades de trabalho; Trabalho em equipe; Habilidades sociais; Pedagogos.

\section{Introducción}

Actualmente, la sociedad se encuentra inmersa en un proceso de cambio dentro de la era de la información, donde esta última, en un contexto de producción, distribución y de gestión, ha revolucionado las circunstancias económicas, comerciales, políticas, e incluso a nivel de comunicación cultural mundial (Vázquez, 2001), implicando así un nuevo modo de actuación y otra forma de vida en general. Esta globalizada sociedad de la que somos miembros activos se contextualiza dentro de un espacio temporal donde el conocimiento y el aprendizaje continuo cobran especial protagonismo, siendo la forma más común de transmisión de cultura la educación formal, aquella que se da en la escuela, con una metodología y unos contenidos establecidos (García \& Belmonte, 2020). "El horizonte educativo en el que nos encontramos inmersos demanda estrategias de enseñanza actualizadas, innovadoras y atractivas” (Bernárdez et al., 2020, p.850). Por ello, este escenario es idóneo para propiciar los rápidos y efímeros cambios, que con frecuencia son inesperados, y los cuales exigen una mayor capacidad de adaptación al medio, imponiendo como requisito el ser capaces de hacer frente a la incertidumbre y la ambigüedad que genera. Esta necesaria adecuación a las demandas que van surgiendo paulatinamente, precisa sobre todo de un desarrollo de estrategias básicas y esenciales de aprendizaje por parte del alumnado (Bordas \& Cabrera, 2001).

"Un profundo proceso de cambio viene acaeciendo estos últimos años en el sistema pedagógico" (Mirete et al., 2020, p.186). Dado que la potenciación de las competencias que permitieran al estudiante conseguir un adecuado desarrollo para ejercer una profesión concreta durante toda su vida laboral, según Ruiz Ruiz (2010), fue uno de los pilares primordiales del proceso de Convergencia Europea y las políticas del Espacio Europeo de Educación Superior (EEES), el presente trabajo de investigación hace referencia al planteamiento de un Programa de intervención centrado en el estudio de las competencias más significativas que actualmente son requeridas por el mundo laboral a los jóvenes de nuestra sociedad.

Estas aceleradas y radicales transformaciones que ha generado la globalización en la sociedad no dejan de afectar a la institución académica y, en particular, a la educación superior, en el conocido como ciclo del conocimiento (Ferro, 2000). Hace apenas unos años, dentro del contexto de globalización económica del pasado siglo XX y durante la revolución tecnológica, las organizaciones e instituciones junto con el concepto de trabajo en sí, se han ido reconfigurando, y lógicamente, el perfil de competencias exigido a jóvenes y trabajadores, también. La sociedad, progresivamente con el cambio, va requiriendo profesionales competentes en ámbitos cada vez más trasversales, y nos encontramos ante la necesidad de poseer altas capacidades de adaptación y actualización. Se reclaman personas versátiles, capaces no solo en el círculo cerrado de su ámbito laboral, sino con actitudes de trabajo en grupo, capacidad de adaptación, flexibilidad, entre otros. No estamos únicamente frente al saber, o saber hacer, sino ante el querer, saber ser y también estar (Talavera \& Pérez-González, 2007). De esta manera, la educación, tanto fomal como no formal (García \& Belmonte, 2020) y las instituciones educativas no representan un agente ajeno, sino todo lo contrario. Se ven afectadas por todas estas transformaciones y juegan un papel protagonista, que les exige replantear sus relaciones con la sociedad (De Vargas et al., 2005). 


\section{La educación por competencias en el marco del EEES}

Ya en los años veinte se mencionaba el tema de las competencias en los círculos pedagógicos (Del Pino, 1997) y en los años treinta se definía como el conjunto de los conocimientos, cualidades, capacidades, y aptitudes que permiten discutir, consultar y decidir sobre lo que concierne al trabajo. Suponiendo conocimientos razonados, ya que se considera que no hay competencia completa si los conocimientos teóricos no son acompañados por las cualidades y la capacidad que permita ejecutar las decisiones que dicha competencia sugiere. No es hasta los años setenta cuando se empieza a tomar en consideración este término. McClelland (1973) vaticinó que exámenes académicos no aseguraban el éxito en el trabajo ni en la vida, por lo que precisó necesario buscar otras variables predictoras. Dichas variables fueron denominadas competencias, y definidas como características esenciales de la persona, que justifican su rendimiento eficiente en el trabajo (Molano, 2013). Desde entonces el interés del mundo organizacional por el tema competencial no ha hecho más que crecer incesantemente.

La noción de competencia, tal como es empleada en relación al mundo laboral, se sitúa a caballo entre los saberes y las habilidades concretas, siendo esta inseparable de la acción, pero exigiendo a la vez conocimiento. Se trata así de un conjunto de propiedades en continua modificación y cambio, que deben ser sometidas a la prueba de la resolución de problemas concretos en situaciones de trabajo que impliquen ciertos márgenes de incertidumbre y complejidad técnica (Gallart \& Jacinto, 1995).

El reto empresarial y universitario planteado a las instituciones es generar valor a través de la creación del conocimiento. Esto hace necesario una redefinición de los principios y teorías que han de orientar el diseño, la gestión y el funcionamiento de las universidades e instituciones vinculadas con el conocimiento en general (Rodríguez et al., 2005), pero con una buena adaptación al cambio se hará frente a las desavenencias, procurando preservar el verdadero valor educativo y garantizando una función social de calidad (Belmonte \& Bernárdez, 2020).

La constitución del EEES ha supuesto un complejo proceso de transformación para la Universidad, donde la organización de la enseñanza gira en torno a un modelo de formación académica centrado en el aprendizaje de los estudiantes (Fidalgo \& García, 2008; Guth, 2006). En estos últimos años, las universidades europeas han ido implantando este sistema común de titulaciones y créditos, lo que ha creado un nuevo escenario que provoca y provocará muchos cambios en la universidad, ya que todos ellos implican una profunda transformación de la educación universitaria, que ha de tomar la competencia profesional como referente de la formación y adoptar también unos métodos docentes diseñados para conseguir que los estudiantes puedan responder a las necesidades de la sociedad y de un mercado de trabajo cada vez más competitivo y sin fronteras. Según Smeby y Trondal (2005), la colaboración internacional para la docencia o para la investigación está experimentando un incremento progresivo bastante importante en toda Europa. Estos cambios fraguados en el marco del proceso de convergencia europea favorecen un notable incremento de la atención dirigida al desarrollo de estrategias transferibles y utilizables para el aprendizaje a lo largo de la vida, y para el empleo (National Commi $\neg$ tee of Inquiry into Higher Education, 1997).

Principalmente, esta construcción de un modelo de formación por competencias se ve justificada en la indiscutible necesidad de un mayor acercamiento entre la universidad y la empresa (Garraway et al., 2011; Ramos \& Fernández-Esquinas, 2012; Schilling \& Klamma, 2010) para asegurar la transferencia del conocimiento, de acuerdo con las nuevas necesidades sociolaborales actuales y emergentes. Como consecuencia de ello, en los títulos, es imprescindible que las competencias que los estudiantes han de adquirir estén formuladas de forma que sean fácilmente identificables, a lo largo de su periodo formativo (Martín del Peso et al., 2013), ya que estas van a configurar su futuro perfil profesional.

Tal y como defiende Delors (2013), en este siglo XXI se plantean una serie de exigencias a la educación características por su casi contradictoria singularidad. Por un lado, se deben transmitir conocimientos teórico-técnicos de forma masiva y eficaz, para adecuarlos a las demandas de las competencias del futuro. Mientras que por otro lado se deben reforzar 
las pautas para no perder el rumbo en el mar de información que abruma nuestra sociedad. De este modo, la justificación de este modelo de formación por competencias se centra en la necesidad indiscutible de un mayor acercamiento entre la universidad y la empresa (Belmonte et al., 2020; Etzkowitz et al., 2000; Garraway et al., 2011; Laursen \& Salter, 2004; Marginson \& Rhoades, 2002; Ramos \& Fernández-Esquinas, 2012; Schilling \& Klamma, 2010; Timoštšuk \& Ugaste, 2010; Valo, 2000) para asegurar la transferencia del conocimiento, de acuerdo con las nuevas necesidades sociolaborales actuales y emergentes. Como consecuencia de ello, se está produciendo una considerable expansión de Grados y Postgrados para dar respuesta a dichas necesidades (Kirby, 2011) y en el diseño de los planes de estudios universitarios, uno de los aspectos más relevantes a tener en cuenta es la identificación de las competencias que los titulados han de adquirir a lo largo de su periodo formativo (Martín del Peso et al., 2013), las cuales, van a configurar su futuro perfil profesional.

El inicio de un modelo de docencia centrado en el proceso de aprendizaje del estudiante, que facilite a su vez desarrollar las competencias necesarias para el futuro profesional del mismo, fue uno de los principales retos del proceso de Convergencia Europea. Así, las competencias propias de cada perfil profesional de Grados y Postgrados se han establecido como el esqueleto referencial para la elaboración de cada titulación y de las asignaturas que las conforman, con el fin de conseguir dar cobertura a las necesidades de una sociedad cambiante en la que el éxito profesional viene determinado por múltiples indicadores (Mora, 2008). Así pues, las ventajas que trae consigo este nuevo modelo de formación por competencias abarcan desde el logro del aprendizaje activo de los estudiantes, hasta la posibilidad de interrelacionar disciplinas, aplicar lo aprendido de forma eficiente en un contexto previamente establecido, y entre otras cosas, según Zabala y Arnau (2007), facilita que el alumno parta de situaciones y problemas reales. Por ello, el trabajo por competencias implica la configuración de la profesionalidad de los estudiantes, promoviendo el cambio hacia su capacitación y la cualificación (Hernández et al., 2005).

Pero esta incorporación de las competencias a la formación debe superar arduas barreras como la enseñanza memorística que lleva implícita la dificultad de aplicar los conocimientos a la vida real (Zabala \& Arnau, 2007). La formación basada en competencias implica un cambio radical en la concepción del proceso de enseñanza-aprendizaje en la universidad y tal y como señala López Ruiz (2011, p. 291) "supone cambiar de un docente transmisor de conocimientos a un profesor-tutor que guía, facilita y supervisa la adquisición de competencias por parte del alumnado. En paralelo, los estudiantes han de modificar su tradicional papel pasivo por uno más activo, participativo e «indagativo»". Según Fitzmaurice (2010), la formación por competencias da un paso más, y sobrepasa la mera visión mecanicista de la enseñanza, ya que se completa con la asimilación de estrategias que integran la excelencia, la complejidad y la integridad.

\section{El grado en Pedagogía de la Universidad de Murcia}

El título de Grado en Pedagogía de la Universidad de Murcia se comienza a diseñar partiendo de una dilatada experiencia de la implantación de la Licenciatura en Pedagogía durante más de tres décadas. El interés académico, científico y profesional de este título es reseñado en varios estudios sobre de la demanda y la inserción laboral de los titulados universitarios. A su vez, datos procedentes de otros informes acerca de proyecciones del mercado laboral justifican la pertinencia del título en relación con perfiles profesionales ya consolidados, o con gran potencial de desarrollo futuro. Asimismo, también destacados referentes externos fundamentan el interés social de este título de grado, como son los providentes de documentos relativos a sistemas, contextos y programas educativos y formativos (González \& Wagenaar, 2003) en los que se constata la importancia de la educación y la formación como sector en auge. Todo ello unido a una rápida expansión, diversificación y profesionalización del pedagogo. De hecho, la formación del profesorado, el desarrollo profesional continuo y la formación de formadores vienen siendo claros objetivos estratégicos de la mejora de la calidad y la eficacia de los sistemas educativos y formativos en la Unión Europea.

El interés social, académico, científico y profesional del título de Pedagogía se apoya en varios estudios acerca de la 
demanda y de la inserción laboral de los titulados universitarios. Asimismo, datos de estrecha relación con el mercado laboral justifican la pertinencia del título en relación con múltiples campos o perfiles profesionales, unos ya consolidados y otros con un alto potencial de desarrollo futuro.

La finalidad fundamental del Grado en Pedagogía es formar profesionales con amplios conocimientos y saberes acerca de las disciplinas relacionadas con la educación y la formación, que sean capaces de liderar proyectos y adaptarse a contextos de desarrollo personal, social y profesional, complejos y en evolución, presentes en los espacios más importantes de la vida educativa y su actividad profesional, como son la comunidad educativa en general, las organizaciones y servicios educativos y formativos, y la formación y desarrollo profesional, laboral y ocupacional en todos sus ámbitos.

Este título pretende preparar en los cuatro años del grado, a profesionales en el campo de la educación, formándolos para que puedan hacer frente a los continuos problemas que surgen en la actividad educativa o formativa, y para que sean capaces de dar respuesta a nuevas demandas dentro de los diferentes contextos, desde los distintos perfiles profesionales atribuibles al pedagogo, como formador de formadores, formador de personas en ámbitos socio-educativos, educador para la igualdad, gestor de organizaciones y procesos, asesor pedagógico, orientador escolar, profesional y laboral, diseñador pedagógico de procesos y recursos, y evaluador de organizaciones, procesos y recursos. Así pues, se hace evidente la importancia de dicho título, dada la alta proyección de la pedagogía en sectores de ocupación en crecimiento, específicamente en lo relativo a las necesidades permanentes de formación inicial y a lo largo del ciclo vital (educación y formación reglada, ocupacional y continua), tan demandados actualmente.

\section{El Problema del Desempleo en España Después de la Universidad}

Desde que en 2008 España se viera acuciada por una intensa crisis económica ha sido uno de los países más afectados de toda Europa. El paro juvenil ha sido una de las principales preocupaciones, siendo esto un agravante para la situación actual de los jóvenes, que ya de por sí son un colectivo con dificultades para entrar en el mundo laboral. Muchos de ellos después de estar varios meses o años en el paro han terminado abandonando la búsqueda de empleo. La preocupación en los jóvenes titulados de encontrar un empleo que tenga que ver con su titulación universitaria es algo latente (Jiménez-Vivas, 2009). Años atrás, el tener un título universitario garantizaba relevancia tanto social como económica (Gaio, 2005), pero esta afirmación ha quedado completamente obsoleta.

La fuga de cerebros se ha convertido en España casi en la única solución para encontrar empleo. Muchos de nuestros universitarios mejor formados se marchan a buscar un futuro mejor, como hacían sus abuelos tiempo atrás. Esto genera en los jóvenes mucha frustración, ya que no ven ninguna recompensa en sus años de esfuerzo. Los que consiguen trabajar en España, lo hacen a tiempo parcial, la gran mayoría y por un sueldo muy bajo, lo que termina conllevando a que no se pueden independizar (Santos, 2013). Como el trabajo no es asequible en España actualmente, nuestros egresados se dedican a formarse años y años, para intentar encontrar un mejor futuro. Lo que ha conllevado a la sobrecualificación de los universitarios españoles, que según las estadísticas se sitúa en el 22\% frente al 13,2\% de la OCDE (García-Montalvo, 2009). Aunque la otra cara de la moneda es que debido a la crisis económica muchos jóvenes no pueden afrontar los gastos que supone estudiar (Abril et al., 2008). El informe "Juventud necesaria: consecuencias económicas y sociales de la situación del colectivo joven" afirma que el 30\% de los jóvenes están en riesgo de exclusión social (Díez-Prieto, 2015). Con consecuencias para los jóvenes que llegan incluso a impedirles formar su propia familia (Weller, 2007).

Es cierto que cada vez se invierte más en educación, pero es importante para el gobierno que la educación superior se adapte a las necesidades del mercado de trabajo (Jiménez-Vivas, 2009). Ya que los tiempos están cambiando, la universidad debe hacerlo también. Se debe renovar y formar a los jóvenes con nuevas capacidades, flexibilidad y autonomía. El mercado de trabajo necesita gente emprendedora (Solé et al., 2007), con competencias transversales, que se capaz de adaptarse a las 
transformaciones que nos acucian. Por eso la universidad debe ser de calidad, estar al tanto de las necesidades sociales y las evoluciones del mundo laboral, enseñando las competencias demandadas y exigidas en ese momento actual, sin olvidar el futuro (Jiménez-Vivas, 2009).

La competitividad de las empresas en la actualidad depende en cierta medida, de la inversión en capital humano que las mismas estén dispuestas a hacer. En pleno siglo XXI, la contratación, el desarrollo y el mantenimiento de trabajadores cualificados resulta crucial para el crecimiento y desempeño empresarial. Según Catalano et al. (2004), la garantía de que los empleados sean competentes y por tanto puedan llevar a cabo su trabajo de manera eficaz, beneficia tanto a la empresa en sí, como a la sociedad en general.

El Servicio Regional de Empleo y Formación (SEF) es un organismo autónomo con la finalidad de realizar una orientación para el empleo estable y de calidad, a través de actividades de fomento, formación para el empleo y de intermediación en el mercado laboral, en el ámbito territorial de la Comunidad Autónoma de Murcia.

La misión del SEF está dirigida a ayudar a los trabajadores o desempleados para la obtención de un puesto de trabajo digno, favorecer la promoción laboral y el reciclaje de los trabajadores, asegurando la igualdad de oportunidades entre hombres y mujeres en el empleo. Tiene como objetivos la modernización del sistema de atención a los demandantes de empleo y la incorporación de las nuevas tecnologías. Por otra parte, el SEF unifica los recursos y servicios regionales relacionados con el empleo. Coordina su actuación con el resto de Administraciones Públicas, y se constituye como vínculo de participación de los Agentes Sociales en la planificación y diseño de estrategias para el empleo y desarrollo. Cuenta con dos áreas funcionales: Área de empleo y Área de formación para el empleo. Además, el Observatorio Ocupacional está encargado de la obtención y mantenimiento de un banco de datos para conocer necesidades y evolución del mercado laboral.

Los servicios que ofrecen las oficinas del SEF:

- Registro y Clasificación como demandantes de empleo.

- Oferta de colocación adecuada.

- Información sobre Medidas de Fomento de Empleo (autoempleo, cooperativismo, sociedades laborales, etc.).

- Cursos de formación profesional para el empleo.

- Orientación para el empleo.

- Análisis de calificación profesional y de las necesidades de formación.

- Ofertas y demandas de empleo en otros países de la Unión Europea, así como su tramitación.

En este proyecto se ha destinado una serie de actividades para la autonomía para la búsqueda activa de empleo, siendo objetivo primordial del servicio de orientación. Se pretende así, combatir problemas actuales en España que influyen en el desempleo juvenil actual: alta tasa de abandono escolar, escasa cualificación o sobrecualificación de los estudios, escasa relevancia de la formación profesional de grado medio, nivel bajo de idiomas extranjeros, muchos contratos a tiempo parcial o temporal, difícil acceso al mercado laboral, hace falta mejorar el autoempleo.

El objetivo del proyecto es diseñar un programa de intervención con el propósito de informar y formar sobre las competencias laborales. La finalidad es la facilitación de la contratación laboral de jóvenes, allanando la transición educaciónmercado laboral, mejorando la empleabilidad de los jóvenes, aumentando la calidad y la estabilidad del empleo joven, igualdad de oportunidades y fomentando el espíritu emprendedor.

\section{Metodología}

El proyecto se desarrolla bajo una metodología de aprendizaje y servicio, propia del siglo XXI (Carbonell, 2015), la cual es considerada hoy en día un foco de interés pertinente y relevante en la investigación educativa (García \& Cotrina, 2015). Esta permite vislumbrar propuestas educativas en el escenario de los nuevos entornos de aprendizaje en relación, que precisan 
ser considerados en el marco de los nuevos retos educativos (Zarzuela \& García, 2020).

Este proyecto comenzará con una charla sobre las competencias laborales. Una de las orientadoras tratará el tema de las competencias laborales transversales, su importancia en el mundo laboral actual, cuáles son las más demandas por las empresas, datos reales sobre la falta de competencias en los jóvenes españoles, el panorama de empleo en España y la finalidad de este proyecto.

Actividad 1: Toma de decisiones.

Objetivo: Asumir el valor e importancia de tomar decisiones.

Desarrollo: Los jóvenes deben responder a estas 4 preguntas claves en el proceso de orientación: ¿Quién soy?, ¿Dónde estoy?, ¿A dónde voy?, ¿Qué puedo hacer?

Con ellas se pretende que reflexionen sobre ellos mismos y los objetivos que se plantean para el futuro profesionalmente. Finalmente leerán cada uno en voz alta la respuesta a la pregunta ¿A dónde voy? y ¿Qué puedo hacer?

Duración: 40 minutos

Actividad 2: Trabajo en equipo "El juego de la NASA".

Objetivo: Descubrir que las decisiones tomadas en equipo son más acertadas que las decisiones de forma individual, y que el trabajo en equipo, en general, es más eficaz que el trabajo individual.

Desarrollo: Este juego versa sobre una nave espacial que tiene problemas, y se debe elegir entre 15 elementos de supervivencia, en orden de importancia. A continuación, se deben establecer primero individualmente los objetos que aparecen en el cuadro por orden de importancia para ellos, luego formarán grupos de cuatro personas y deben debatir para volver a ordenar los objetos de nuevo por orden de importancia, una vez terminado en pequeño grupo, todos los participantes tendrán que ponerse de acuerdo para volver a hacer la lista de los objetos. Para finalizar la orientadora leerá el resultado justificando los motivos de cada objeto y su importancia para la supervivencia.

Duración: 45 minutos.

Actividad 3: Caso práctico.

Objetivo: Reflexionar sobre el trabajo en equipo.

Desarrollo: En grupos de cinco personas se leerá un caso práctico y reflexionarán sobre el trabajo individual o el trabajo en equipo y cuál de ellos tiene más ventajas. Cada portavoz del grupo leerá al resto de la clase sus respuestas justificándolas.

Duración: 25 minutos.

Actividad 4: Casos educativos.

Objetivo: Proponer soluciones a casos concretos.

Desarrollo: En grupos de cuatro personas se deberá leer y argumentar posibles soluciones a los casos educativos que aparecen. El primer caso está relacionado con problemas del grupo de clase con el profesor de matemáticas. El segundo caso hace referencia a los trastornos alimenticios de una alumna. El tercer caso, una discusión entre padres en la puerta del colegio. El cuarto caso, un niño inmigrante que no hace nada en clase. Deben poner en práctica sus conocimientos pedagógicos para resolver los conflictos planteados, llegando a un acuerdo entre compañeros, ya que una de las competencias del pedagogo es el trabajo en equipo. 
Actividad 5: Video "Trabajo en equipo, ese no es mi problema".

Objetivo: Descubrir los conflictos que conlleva el no trabajar en equipo.

Desarrollo: Tras la visualización del vídeo que va orientado a la importancia de la asunción de nuestra responsabilidad en el trabajo, y la facilitación de las tareas si se llevan a cabo en equipo, la orientadora abrirá un debate para que los participantes den su opinión acerca del video, sus sensaciones, propuestas de mejora, comportamientos observados en el vídeo, moraleja del video.

Duración: 20 minutos

Actividad 6: Habilidades sociales "Vendiendo imposibles".

Objetivo: Mejorar la comunicación verbal.

Desarrollo: Potenciar la capacidad para vender cualquier artículo que se precie, por muy extravagante o imposible de vender que parezca, con el fin de aumentar la autoestima y confianza en las propias capacidades para la venta de uno mismo y desarrollar la capacidad de improvisación. El orientador prepara, con anterioridad a la sesión, una lista con el nombre de diferentes artículos para la venta, como, por ejemplo: zapatos para elefantes, pijamas para osos, patines para canguros, motos para tortugas, cojines para camellos, cortaúñas para gatos, gomina para erizos, calcetines para pulpos, gafas para piojos, etc. Se empieza la actividad explicando que cada uno va a tener que salir del aula para luego volver a entrar "metido" en el papel de vendedor; que en un minuto intente convencer al resto de que su producto es el mejor. Cada participante presenta su producto durante el minuto de intervención. Cada compañero puede hacer preguntas, como cliente potencial. Al final, cada uno comenta la experiencia y el orientador refuerza la participación por haber sido capaces de vender estos artículos, presentando esta actitud como clave para participar con éxito en una entrevista de trabajo.

Duración: 30 minutos

Actividad 7: No puedo hablar con nadie de mi suerte.

Objetivo: Trabajar las relaciones sociales, haciendo una reflexión sobre las cosas buenas que nos gustan compartir con los demás.

Desarrollo: Se presenta una historia en la que un sujeto no tiene amigos para compartir su suerte.

"Una persona estaba disfrutando de un momento de suerte, tenía un número de lotería en el que había recibido unos 4.000.000 de euros, pero se encontraba en un lugar donde no conocía a nadie. Pensó en acudir a uno de los mejores restaurantes de esa ciudad y después ir a unos grandes almacenes donde comprarse ropa nueva, un buen reloj; pero finalmente se hizo de noche y tuvo que ir al hotel donde se hospedaba, tampoco había nadie y no encontró con quien compartir esa suerte"

Se organiza un debate en el que dos grupos intentan defender posturas contrarias: uno de ellos argumentará en torno a que la suerte es suerte, aunque no se pueda compartir. El otro analizará la historia y razonará que la suerte, cuando no se tiene a nadie a quien contárselo, es un poco menor, todos deberán hablar. Por último, una reflexión grupal ayudará a ordenar estas ideas, solicitando una postura razonada del gran grupo en la que se pueda llegar a conclusiones como ésta: es un elemento muy importante en nuestra vida, el otro; esa persona que tenemos siempre a nuestro lado y que no valoramos su peso.

Duración: 20 minutos

Actividad 8: El lenguaje del cuerpo.

Objetivo: Analizar la importancia del lenguaje no verbal en el proceso de comunicación. 
Desarrollo: Se muestra la importancia del lenguaje del cuerpo, con una exposición como ésta: una persona puede decidir no hablar o ser incapaz de comunicarse verbalmente, y aun así seguir emitiendo mensajes por medio de su cara y de su cuerpo. El orientador solicita la participación de dos miembros del grupo para realizar la simulación de una conversación muda, es decir, sin que durante la misma se hable ni emita ningún tipo de sonido. Cada participante adoptará un rol, representando uno el papel de persona con bajas habilidades sociales, y el otro el de una persona con habilidades sociales muy desarrolladas.

Se conceden diez minutos para preparar la simulación; mientras se le dan instrucciones al resto del grupo para que esté atento a la postura corporal, gesticulación, contacto visual, expresión facial, distancia, proximidad y contacto físico. Se realiza la simulación durante cinco minutos. El gran grupo analiza los rasgos observados sobre altas o bajas habilidades sociales. Finalmente, el orientador informa al grupo de los rasgos del lenguaje no verbal que diferencia a las personas con altas y bajas habilidades sociales.

Duración: 30 minutos

Actividad 9: Autonomía para la búsqueda activa de empleo "Entrevista de trabajo".

Objetivo: Conocer las claves para realizar una buena entrevista de trabajo.

Desarrollo: La orientadora explicará unas pautas a seguir en una entrevista de trabajo, tales como: preparar la entrevista, manejar la ansiedad, conocer tus puntos fuertes y débiles, saber venderte, contestar de forma positiva, vocabulario rico, contestar preguntas difíciles, etc. Además de las conductas básicas durante la entrevista: contacto visual, tono emocional, claridad en la voz, fluidez verbal, motivación, seguridad, etc.

Individualmente los alumnos prepararán una posible entrevista de trabajo y por parejas, la simularán. Uno de ellos hará de entrevistar y otro de entrevistado. Al finalizar, toda la clase debe opinar sobre los fallos de la entrevista, para mejorarlos.

Duración: 60 minutos

Actividad 10: Videocurriculum y carta de presentación.

Objetivo: Conocer herramientas para buscar empleo y aprender a elaborar una carta de presentación.

Desarrollo: La orientadora proporcionará información sobre el videocurriculum, con una breve introducción, explicación de qué es un videocurriculum, sus ventajas e inconvenientes, como hacer un videocurriculum paso a paso, consejos, ejemplos de videocurriculums en la red, y como puedes hacer un videocurriculum web.

Los participantes deben crear su propio videocurriculum para su posterior visualización en grupo. El video debe durar tres minutos y debe ser original. Entre todos elegirán el mejor videocurriculum. Además, la orientadora dará las pautas necesarias para elaborar la carta de presentación, qué debe ponerse en cada apartado, estructura, contenido, consejos. Cada uno tendrá que elaborar individualmente una carta de presentación siguiendo las pautas y entregársela a la orientadora.

Duración: 60 minutos

\section{Evaluación}

La evaluación, entendida como elemento integrante del proceso de enseñanza aprendizaje y no como factor formativo y controlador (García-Sanz, 2011), acorde con la metodología y objetivos del proyecto, será continua, individual e integradora, llevada a cabo como proceso y no como actuación puntual.

Los resultados esperados a corto plazo es conseguir que los participantes sean informados e interioricen la importancia de tener competencias laborales transversales a la hora de desarrollar un empleo, que las competencias de trabajo en equipo y habilidades sociales son necesarias para desarrollar la profesión de pedagogía, y que adquieran autonomía para buscar un empleo de forma autónoma y eficaz. Los resultados que se esperan obtener a largo plazo es que las personas 
implicadas desarrollen las competencias transversales en su puesto de trabajo como pedagogos.

Este proyecto tiene como base la evaluación continua, que se hace a lo largo del mismo. La técnica utilizada para evaluar es la observación. Una observación directa sobre el proceso de mejora de los participantes y si el proyecto está teniendo utilidad.

Para la elección de los instrumentos de la evaluación se tiene fundamentalmente en cuenta la observación directa sistemática del orientador y mediador de las actitudes y trabajo desarrollado por los participantes. El contraste entre los objetivos planteados y el grado de conocimientos, destrezas y habilidades adquiridas. La implicación y participación de los destinatarios en todo el proceso a través de la autoevaluación individual, grupal y en gran grupo.

En este espacio de reflexión crítica sobre la práctica y las acciones del curso, se analizarán aspectos como la correspondencia entre las actividades programadas y los objetivos propuestos, la respuesta a las necesidades formativa de los participantes, las habilidades docentes de los orientadores, las técnicas, métodos, soportes adecuados y de calidad, el aprendizaje de los participantes en función de los objetivos, el nivel de optimización de los recursos, o la duración, horarios y organización adecuada. A lo largo del proyecto se generará un proceso de reflexión y retroalimentación constante entre todo el equipo, con la participación de los destinatarios, teniendo en cuenta las actividades que realizan, comportamiento, participación, motivación. Evaluando el proyecto a través de la recogida sistemática de información mediante actividades individuales y grupales, observación sistemática, seguimiento, cuestionario de satisfacción. Como herramientas de evaluación se utilizarán las actividades realizadas individualmente por los participantes, como las cartas de presentación, donde se valorará, la estructura de la carta, contenido, información, claridad, presentación. Además del videocurriculum, donde se valorarán aspectos como la originalidad, contenidos, estructura, diseño, duración.

Al finalizar el proceso se hará una evaluación sumativa, para obtener resultados de satisfacción de los participantes. Para ello se aplicará un cuestionario, que proporcionará información para conocer el grado de aceptación del proyecto. Este cuestionario recoge aspectos de: organización, contenidos, orientador, metodología, valoración general, con una escala de puntuación de cinco grados.

\section{Consideraciones Finales}

Las diferentes competencias que debe desarrollar los estudiantes de pedagogía han de estar a la altura de las necesidades de la sociedad del S. XXI. Para ello, las diferentes actividades planteadas han de ser consecuentes con las competencias que se deben desarrollar a lo largo de los estudios oficiales. Cómo se ha podido observar, dicha necesidad puede ser cubierta con una gran diversidad de actividades, de las que aquí se ha realizado una pequeña muestra. Finalmente, Se observa como un elemento de importancia la evaluación que se realice, puesto que, como se apuntaba, es espacio de reflexión crítica sobre la práctica y las acciones del curso.

\section{Referencias}

Abril, E., Román, R., Cubillas, M. J. \& Moreno, I. (2008). ¿Deserción o autoexclusión? Un análisis de las causas de abandono escolar en estudiantes de educación media superior en Sonora, México. Revista electrónica de investigación educativa, 10(1), 1-16.

Belmonte, M. L., García-Sanz, M. P., \& Galián Nicolás, B. (2020). Dos perspectivas de una misma meta: pertinencia y nivel de logro de las competencias universitarias. Educar, 56(2), 423-438.

Belmonte, M. L., \& Bernárdez, A. (2020). Respuesta social al estado de aislamiento por coronavirus, percepciones sobre educación. Revista Conhecimento Online, 3, 30-49.

Bernárdez, A., Belmonte, M. L., \& Galián, B. (2020). Microenseñanza y Autoscopia como Elementos de Evaluación Docente, de la Teoría a la Práctica. Revista Meta: Avaliação, 12(37), 848-868.

Bordas, M. I. \& Cabrera, F. A. (2001). Estrategias de evaluación de los aprendizajes centrados en el proceso. Revista Española de Pedagogía, $218,25-48$. 
Carbonell, J. (2015). Pedagogías del siglo XXI: Alternativas para la innovación educativa. Octaedro.

Catalano, A. M., Avolio de Cols, S., \& Sladogna, M. (2004). Diseño curricular basado en normas de competencia laboral: conceptos y orientaciones metodológicas. Cinterfor.

De Vargas, M. R., Barrios, B. J., \& Santiago, L. R. (2005). Competencias laborales y la formación universitaria. Psicología desde el Caribe: revista del Programa de Piscología de la Universidad del Norte, (16), 64-91.

Del Pino, A. (1997). Empleabilidad y competencias: ¿nuevas modas? En M. Ordoñez (coord.), Psicología del Trabajo y Gestión de Recursos Humanos. Barcelona: Gestión 2000, 104-116.

Delors, J. (2013). Los cuatro pilares de la educación. Galileo, (23).

Díez-Prieto, A. D. (2015). La juventud actual en el punto de mira. Educar(nos), (69), 4-7.

Etzkowitz, H., Webster, A., Gebhardt C., \&. Terra, B. R. C. (2000). The future of the university and the university of the future: evolution of ivory tower to entrepreneurial paradigm. Research Policy, 29(2), 313-330.

Ferro, J. (2000). Visión de la universidad ante el siglo XXI. Ediciones Uninorte.

Fidalgo, R. T., \& García, J. N. (2007). Las directrices del Espacio Europeo de Educación Superior en el marco legislativo del sistema universitario español. Aula Abierta, (35), 35-48.

Fitzmaurice, M. (2010). Considering teaching in higher education as a practice. Teaching In Higher Education, 15(1), 45-55.

Gaio, M. (2005). La inserción profesional de titulados superiores desde una perspectiva educativa. Revista Europea de Formación Profesional, (34), $30-44$.

Gallart, M. A., \& Jacinto, C. (1995). Competencias laborales: tema clave en la articulación educación-trabajo. Boletín Educación y Trabajo, 6(2).

García, M., \& Belmonte, M. L. (2020). Educación no formal en los grupos scouts. Revista Educativa HEKADEMOS, $28,43-54$.

García, M., \& Cotrina, M. (2015). El aprendizaje y servicio en la formación inicial del profesorado: De las prácticas educativas críticas a la institucionalización curricular. Revista de Currículum y Formación del Profesorado, 19(1), 1-6.

García-Montalvo, J. (2009). La inserción laboral de los universitarios y el fenómeno de la sobrecualificación en España. Papeles de Economía Española, 119, $172-187$.

García-Sanz, M. P. (2011). Innovaciones orientadas al EEES. Las competencias y su evaluación como elementos de planificación en el marco del EEES. En J. J. Maquilón (coord.). La formación del profesorado en el siglo XXI. Propuestas ante los cambios económicos, sociales y culturales (pp. 63-79). Editum.

Garraway, J., Volbrecht, T., Wicht, M., \& Ximba B. (2011). Transfer of knowledge between university and work. Teaching in Higher Education, 16(5), 529540 .

González, J., \& Wagenaar, R. (2003). Tuning Educational Structures in Europe: Final Report-Phase One. Universidad de Deusto.

Guth, J. (2006). The bologna process: The impact of higher education reform on the structure and organisation of doctoral programmes in Germany. Higher Education in Europe, 31(3), 327-338.

Hernández, F., Martínez, P., Da Fonseca, P., \& Rubio, M. (2005). Aprendizaje, competencias y rendimiento en Educación Superior. La Muralla.

Jiménez-Vivas, A. (2009). Reflexiones sobre la necesidad de acercamiento entre universidad y mercado laboral. Revista Iberoamericana de Educación, 50(1), 4.

Kirby, D. (2011). Strategies for widening access in a quasi-market higher education environment: recent developments in Canada. Higher Education, 62(3), 267-278.

Laursen, K., \& Salter, A. (2004). Searching high and low: what types of firms use universities as a source of innovation? Research Policy, 33 , $1201-1215$.

López Ruiz, J. I. (2011). Un giro copernicano en la enseñanza universitaria: formación por competencias, Revista de Educación, 356, $279-301$.

Marginson, S., \& Rhoades, G. (2002). Beyond national states, markets, and systems of higher education: A glonacal agency heuristic,Higher Education, 43(3), 281-309.

Martín del Peso, M., Rabadán Gómez, A. B., \& Hernández March, J. (2013). Desajustes entre formación y empleo en el ámbito de las Enseñanzas Técnicas universitarias: la visión de los empleadores de la Comunidad de Madrid. Revista de Educación, 360, $244-267$.

McClelland, D. (1973). Testing for competence rather than for intelligence. Amercian Psychologist, 28, 1-14.

Mirete, A. B., Belmonte, M. L., \& Maquilón, J. J. (2020). Diseño, aplicación y validación de un instrumento para Valorar las Actitudes hacia la Diversidad del Alumnado (VADA). Revista Electrónica Interuniversitaria De Formación del Profesorado, 23(2), 185-207.

Molano, C. (2013). La formación y la capacitación fundamento y condición esencial en la gestión de los recursos humanos por competencias laborales. Encuentro Internacional. La gestión de los recursos humanos por competencias laborales, Cali, 1-26.

Mora, J. G. (2008). El «éxito laboral» de los jóvenes graduados universitarios europeos. Revista de Educación, número extraordinario, 41-58. 
Research, Society and Development, v. 10, n. 1, e53210112192, 2021

(CC BY 4.0) | ISSN 2525-3409 | DOI: http://dx.doi.org/10.33448/rsd-v10i1.12192

National Commitee of Inquiry into Higher Education (1997). Higher Education in Learning Society. Stationery Office.

Ramos, I., \& Fernández-Esquinas, M. (2012). Beneath the tip of the iceberg: exploring the multiple forms of university-industry linkages. Higher Education, 64(2), 237-265

Rodríguez, A. S., Fornaris, C. E. M., \& Martínez, C. C. M. (2005). Una mirada a los orígenes de las Competencias Laborales. Ciencias Holguín, 11 (2), 1-14.

Ruíz Ruíz, J. M. (2010). Evaluación del diseño de una asignatura por competencias, dentro del EEES, en la carrera de Pedagogía: Estudio de un caso real. Revista de Educación, 351, 435-460.

Santos, A. (2013). Fuga de cerebros y crisis en España: los jóvenes en el punto de mira de los discursos empresariales. Revista Internacional de Ciencias Sociales, 32, 124-137.

Schilling, J., \& Klamma, R. (2010). The difficult bridge between university and industry: a case study in computer science teaching. Higher Education, 35(4), 367-380.

Smeby, J. C. H., \& Trondal, J. (2005). Globalisation or europeanisation? International contact among university staff, Higher Education, 49, 449-466.

Solé, C., Rubio, S. P., \& Cavalcanti, L. (2007). El empresariado inmigrante en España. Fundación" la Caixa".

Talavera, E. R., \& Pérez-González, J. C. (2007). Formación en competencias socioemocionales a través de las prácticas en empresas. Revista Europea de Formación Profesional, (40), 92-112.

Timoštšuk, I., \& Ugaste, A. (2010). Student teachers' professional identity. Teaching and Teacher Education, 26(8), 1563-1570.

Valo, M. (2000). Experiencing work as a communications professional: Students' reflections on their off-campus work practice. Higher Education, 39, 151179.

Vázquez, Y. A. (2001). Educación basada en competencias. Educar: revista de educación/nueva época, 16, 1-29.

Weller, J. (2007). La inserción laboral de los jóvenes: características, tensiones. Revista de la CEPAL, 92, 62.

Zabala, A., \& Arnau, L. (2007). Cómo aprender y enseñar competencias. Graó.

Zarzuela, A., \& García, M. (2020). Comprendiendo el encuentro entre significados del Aprendizaje y Servicio crítico en contextos comparados. Estudios pedagógicos (Valdivia), 46(1), 57-77. 